\title{
Frame Work on an Injection Molding Rejuvenation Technique for Cable Insulation Failure ${ }^{*}$
}

\author{
ChongWei Lv $v^{1,2, a}$, YongXiang Zhao ${ }^{1,3, b, * *}$, JunGuo Wang ${ }^{1, c}, \quad$ ZhiWei Liu ${ }^{1, d}$ \\ ${ }^{1}$ School of Mechanical Engineering, Southwest Jiaotong University, Chengdu, China \\ ${ }^{2}$ Shenhua Railway Wagon Transportation Company Ltd, Beijing, China \\ ${ }^{3}$ Technology and Equipment of Rail Transit Operation and Maintenance Key Laboratory of Sichuan Province, Chengdu, China
}

\begin{abstract}
Rejuvenation technique on cable insulation failures is a hot topic in improving production benefits for electric related enterprises. Breaking the traditional thought of repair action without extensive former damage in scale of the cable insulation, the proposed technique starts from the local standard geometry elimination on insulation or sheaths around cable insulation failure section(s). And then, one standard injection molding technique is applied for rejuvenation on eliminated insulation no matter of the failure resulting from water tree or local mechanical damage or other modes. By this advancement, an injection molding technique is perfectly developed for rejuvenation on cable insulation failure. Frame work on this technigue is hear introduced and it consists of three branches including injection molding material development, standard injection plastic molding technique, and coded quality approval regularities. Realizability and reliability of the present technigue have been verified by sampling testing to expect to be wide applied in production.
\end{abstract}

\section{Introduction}

Cable is the basic component of electrical equipment which is widely applied in power transmission. Its reliability is often affected by insulation damage. Therefore, effective rejuvenation on the insulation failure is a great of scientific research proposal.

So far, it has been revealed that there are two modes for the cable insulation failure. One is water tree damage in which the cable is used in humid environment. Due to the existence of tiny defects on the surface of cable conductive body, water molecules in the environment are gathered to form water trees under the action of electric field. Water trees continue to develop into electric trees, and the electric trees result in the cable does not work normally [1-3]. The other is local mechanical forced damage in cable transportation processes or abnormal work environments.

In view of water tree damaged failure, a comprehensive method of liquid injection chemistry was developed to try to restore the cable function[4]. In which the rejuvenation fluid was injected into the interface between the cable insulation and the conductor body where water tree damage was existent and a stable and insulating compound was also expected to be formed. It was reported that, if silicone resin solution was used as the rejuvenation solution, the compound of chemical reaction was $\mathrm{SiO} 2$ with particles nearby nanometer scale. And, if Titanic solution was used, the compound was $\mathrm{TiO} 2$ with particles nearby nanometer scale. But it is grateful that the successful rejuvenations appeared only in laboratory not in production. For the mechanical damaged failure, the existent rejuvenation approaches appeared crudely and primarily such as the filler fixation, self-adhesive insulation belt twining and molten material-filling fixation in the damaged locations [5-6].

Modern electrified rail transit is a huge cable network world, including the ultra-high voltage transmission between power plant and railway power substations, the high-voltage transmission between the substations and the train electrical cabinets by pantograph, and the decreasing high-voltage transmission between the cabinets, and equipments such as motors. There are strict requirements of electrical conductivity, environmental safety, ageing resistance and mechanical strength for the cables[7]. To assure high quality repair effect, a new cable repair technique-insulation injection molding rejuvenation technique is now developed by the present team. The frame work is here introduced below.

\section{BASIC CONSIDERATIONS}

\subsection{The existing rejuvenation techniques}

By the above introduction, the existing cable insulation rejuvenation techniques include mainly the liquid injection chemistry for the water tree damaged and the filler fixation, self-adhesive insulation belt twining and molten materialfilling fixation method for the mechanical damaged. These techniques are actually few of used in production. Main causes should be discussed below:

\footnotetext{
alcw0317@126.com b1760465099@qq.com cjg_wang2000@163.com d779894575@qq.com
} 
(1) The liquid injection chemistry technique is actually performed under invisible environments. First, the accurate distribution, size and thickness of water tree erosion are not accurately inspected. Hence it is impossible to accurately control the chemical solution flowing through the interface between the insulation and conductor layer. And also it is difficult to control the liquid injection and the chemical synthesis to obtain proper compound and well eliminate water tree erosion effects. Even if all of these are not controllable, it is difficult to guarantee that the chemical solution does not damage the cable. In addition, after the chemical reaction it is difficult to remove/clean/dry the residual chemical solution on the interface. Therefore, this technique may eventually become a paper technology but isn't applicable.

(2) The repair techniques, including the filler fixation, self-adhesive insulation belt twining and molten materialfilling fixation, for the surface mechanical force damages, can be performed in a crude and primary mode with poor quality because of

1) The damage is uncertain in shape and size. The repair quality is difficult to be controlled. Therefore, this repair action maybe applied for temporary treatment.

2) The cables in use are difficult to avoid the mechanical forces such as bending and tensioning. The repair effects are easy to appear separation between the repair materials and the original materials.

Therefore, the existent liquid injection chemistry technique for treating the failures by water tree erosion should be difficultly applied from laboratory to production. While the techniques filler fixation, self-adhesive insulation belt twining and molten material-filling fixation have poor repair quality for the failures from mechanical forced damages.

\subsection{New ideas and framework of the rejuvenation technique}

To solve the relevant technical problems, our team breaks through the traditional thinking that the maintenance action does not expand the damage of products, and introduces the standardized geometric shape removal of insulation/sheath materials at the cable insulation failure position. In this way, the cable with the same cross-section, whether water tree failure or mechanical damage failure, can be repaired by a set of standard technology.

Based on the new ideas, aiming at the goal of highquality standardized repair, we have developed a set of standardized rejuvenation technology of local injection repair for cable insulation failures. This technique also belongs to mechanical packing fixation technology, but the standardization and systematization distinguish the existing technology, and overcome the difficulties of the existing repair technology, which is composed of the following three aspects.

(a) Repair material matching the original insulating sheath

From the point of view of ensuring the effectiveness of repair, the insulation, electrical conductivity, environmental safety and aging resistance and mechanical properties of the repaired materials should be close to or even slightly better than that of the raw materials. At the same time, it is necessary to avoid the interface damage caused by the affinity and potential difference between the repair materials and the original materials. Therefore, the concept of matching includes the basic homogeneity, the same preparation process and the same insulation performance.

(b) Standardized injection mold and repair process

It contains three levels meaning, and they are interdependent and become a set of systematic cable insulation failure rejuvenation technology. The first is a standardized and standardized local active removal strategy for insulation/sheath of cable with insulation failure. That is, no matter what the cause of cable insulation failure and the difference of failure area size shape, as long as it is worth repairing, the cable insulation/sheath partial removal scheme with standardized geometric morphology can be adopted to realize the standardized repair of cables of the same cross-section type.

The second is the standardized injection mold and repair process. It is a kind of cross-section cable, corresponding to a set of cable local standardized removal of geometric space, and a set of matching to remove geometric space to achieve the purpose of injection molding filling. The third is the standardized injection molding maintenance process. That is to say, a kind of repair material, a set of matching cable to remove space of mold, corresponding to a set of injection molding conditions and environment of injection molding machine and mold temperature machine, it should be a set of process flow and environmental process parameters that can achieve good repair effect.

(c) Standardized repair inspection accreditation rules

Whether a maintenance technology can be applied to production is not a question of whether it can be repaired or not, but whether the maintenance effect can meet the requirements of the production. As mentioned above, the cable belongs to electrified rail transit or power production and transmission, urban power supply and other departments, whose maintenance effect is required by high quality. The cable insulation failure repair technology can be produced and applied only if the standardized process conditions and the maintenance effect are inspected according to the acceptable standardization, and it is proved that the acceptable maintenance technology can be met.

\section{Injection Molding Mould and Process}

\subsection{Technical elements}

As shown in Figure 1, the core technical elements of the rejuvenation technique include the following aspects.

(1) Cable (1) to be repaired: Before repair, it is made by partial removal of $1 \mathrm{a} / 1 \mathrm{~b}$ insulation/sheath of the cable with standardized and standardized insulation failure. Therefore, the factors of cable insulation failure caused by local "water tree defect" or "mechanical damage defect" are effectively eliminated. The local insulation/sheath material with length $\mathrm{L} 1 / \mathrm{L} 2$ and thickness $\mathrm{t} 1 / \mathrm{t} 2$ is removed from the conductor with diameter $\mathrm{d}$. After eliminating the problem 
of insulation failure, $1 \mathrm{c} / 1 \mathrm{~d}$ space is left for cable 1 to be filled.

(2) Inner and outer insulation/sheath material (2 or 3 ) to be repaired: Its function is to match the performance of the original inner and outer insulation/sheath materials. Meanwhile, the material has good physical and mechanical properties, conductive and insulating properties, environmental aging resistance, flame retardant and nontoxic properties, as well as good injection/repair process performance.

(3) Mold (4 or 5) for direct repair of inner and outer insulation/sheath: After the cable (1) to be repaired is installed, the repair space $1 \mathrm{c} / 1 \mathrm{~d}$ to be filled is formed. In addition, the channel $4 \mathrm{a} / 5 \mathrm{a}$ connecting the feeding pipe $8 \mathrm{a}$ of the injection molding machine, and the channel $4 \mathrm{~b} / 5 \mathrm{~b}$ connecting the insulation pipe $9 \mathrm{a}$ of the mold temperature machine are also provided.

(4) Mold (6 or 7) for indirect repair of inner and outer insulation/sheath: There is a space $6 \mathrm{a} / 7 \mathrm{a}$ for the preparation of internal and external insulation/sheath repair film (10 or 11), a channel $6 \mathrm{a} / 7 \mathrm{a}$ for connecting the feeding pipe $8 \mathrm{a}$ of the injection molding machine, and a channel $6 \mathrm{~b} / 7 \mathrm{~b}$ for connecting the insulation pipe $9 \mathrm{a}$ of the mold temperature machine.

(5) Injection molding machine (8): It is mainly composed of plastic injection melting device, melting material feeding pipe $8 \mathrm{a}$ and mold pressure holding device $8 \mathrm{~b}$. It has the functions of automatic adjustment and control of injection capacity, melting temperature, feeding pressure and holding time.

(6) Mold temperature machine (9): It is mainly composed of circulating liquid heating device, circulating hydraulic pipeline $9 \mathrm{a} / 9 \mathrm{~b}$ and temperature control system.

(7) Repair mold (10 or 11) for inner-outer insulation/sheath: The volume of repair mold is $1 \mathrm{c} / 1 \mathrm{~d}$ of the space to be filled in inner/outer layers of the cable to be repaired. Its length a1/a2 is slightly less than the removal length L1/L2 of the inner-outer insulation/sheath material of cable (1), and its thickness $\mathrm{t} 1 / \mathrm{t} 2$ is slightly larger than that of the inner-outer insulation material of cable (1), besides, its width is perimeter $(\mathrm{d}+\mathrm{t} 1) /(\mathrm{d}+\mathrm{t} 2)$.

(8) Repair adhesive for soft insulating material (12): It has good insulation performance, and good affinity with the inner and outer insulation/sheath repair and raw materials.

\subsection{Injection molding process}

After effective partial removal and insulation problem treatment, the part of cable insulation failure then becomes cable (1) to be repaired, and it is installed on the direct repair mold (4 or 5) in turn, which forms the cable repair space $1 \mathrm{c} / 1 \mathrm{~d}$ to be filled. It is necessary to connect mold temperature machine (9) and mold (4 or 6) correctly and control mold temperature effectively. Meantime, it needs to connect the injection molding machine 8 and the mold (4 or 5) channel and control the injection molding parameters effectively. In addition, the qualified inner and outer insulation/sheath repair materials are sent to the space $1 \mathrm{c} / 1 \mathrm{~d}$ to be filled in molten state through the feeding pipe of the injection molding machine, and the $1 \mathrm{~g}$ shape is obtained by the treatment of soft insulation material repair glue (12).

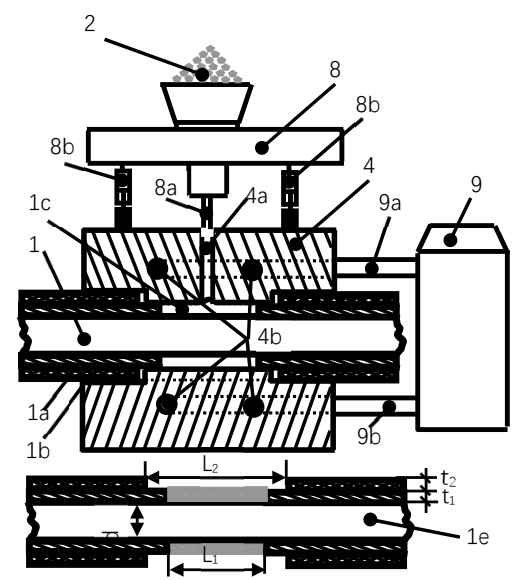

a. Inner insulation injection molding maintenance



b. Injection molding maintenance of outer sheath

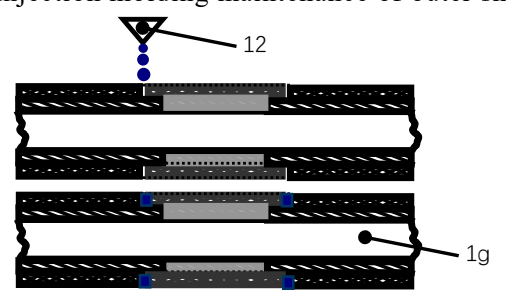

c. Surface treatment after injection molding

Fig1. Directive injection plastic rejuvenation technology for inserting spaces of eliminated cable insulation and sheath layers around failure location

When the inner/outer insulation or sheath repair materials do not have good injection molding process, some indirect injection molding process is needed to achieve repair. As shown in Fig. 2, the insulation failure part becomes the cable (1) to be repaired after effective treatment of the insulation problem. When the repair material ( 2 or 3 ) of inner/outer layer insulation/sheath, or one of them is impossible to repair by direct injection molding, the space 1c/1d to be filled in cable (1) shall be pasted with repair film (10 or 11), according to the principle of the same circumference and volume. Then it is placed in the direct repair mold (4 or 6), the mold temperature machine (9) is connected with the mold (4 or 6 ), and the pressure holding and time control functions of the injection molding machine (8) are applied to make the repair film (10 or 11$)$ fill the space $1 \mathrm{c} / 1 \mathrm{~d}$, so as to make the cable form $1 \mathrm{e} / 1 \mathrm{f}$ in turn, and make the repair glue (12) to get $1 \mathrm{~g}$ shape. 


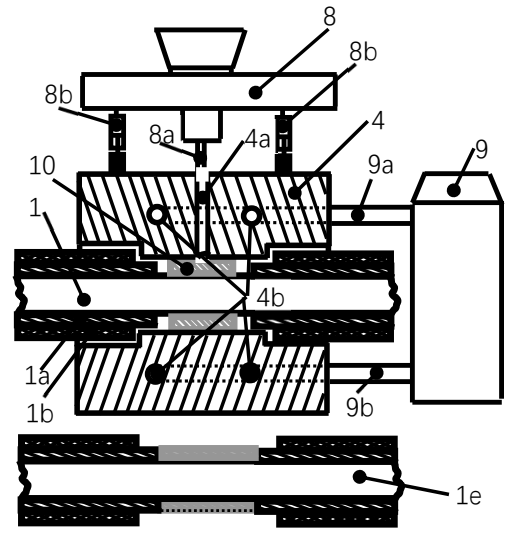

(a) Extrusion repair of inner insulating film

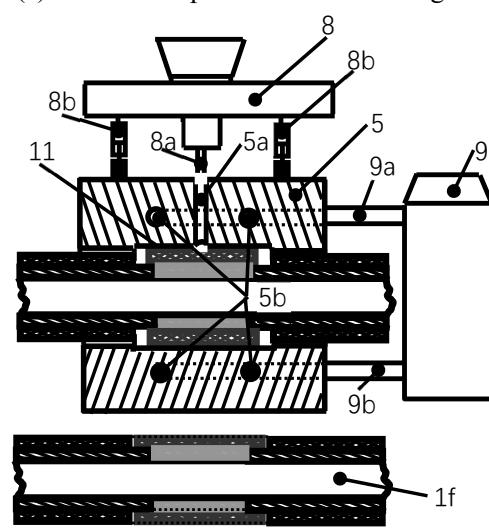

(b) Extrusion repair of outer sheath film

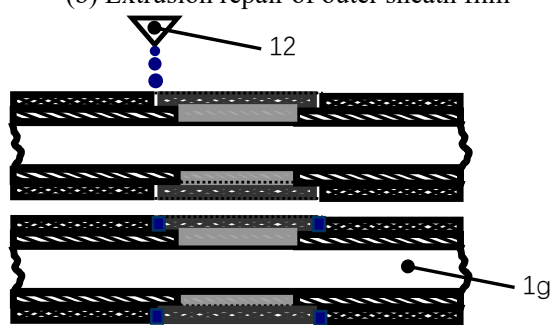

(c) Surface treatment after extrusion

Fig2. Indirective injection plastic rejuvenation technique for inserting spaces of eliminated cable insulation and sheath layers around failure location

\section{Conclusions}

(1) The developed local injection rejuvenation technology for cable insulation failure includes injection repair materials matching the original insulation/sheath material, standardized repair equipment and process, and standardized repair quality inspection and approval rules. They are interrelated and form an innovative cable rejuvenation technology system.

(2) This technology introduces the standardized geometric shape local excision of cable failure parts, so that all kinds of failure of a cable can be completed by a standardized injection molding process. It is a comprehensive and reliable production technology of cable insulation maintenance, which breaks through the traditional passive repair behavior.

(3) The proposed technique of local injection rejuvenation of cable insulation failure is put into use and expected to be widely applied in production.

\section{Acknowledgment}

The work is supported by National Basic Research Project of China (973 Program, 2015CB654801), Scientific and technological innovation project of Shenhua Group (HCKY-2016-13), and Sichuan Youth Science and technology innovation team project (2017TD0017).

\section{References}

1. S. Boggs, J. Densley and J. Kuang, "Mechanism for impulse conversion of water trees to electrical trees in XLPE," IEEE Trans. on Power Delivery, vol. 13, pp. 310-315, February 1998.

2. R. Ross, "Inception and propagation mechanism of water treeing," IEEE Transactions on Dielectrics and Electrical Insulation, vol. 5, pp. 660-680, May 1998.

3. R. Ross and J. Smith, "Composition and growth of water trees in XLPE," IEEE Transactions on Electrical Insulation, vol. 27, pp. 519-533, March 2002.

4. Z. Li, H. Deng and Z. Wu, A liquid injection technology for rejuvenation high voltage cable, China: CN201220376115, 2012.

5. J. Wang and L. Ma, A cold sticky rubber band for rejuvenation mining cable, China: CN2272624Y, 1996.

6. H. Teng, A equipment for rejuvenation damaged insulation of cable with supporting. China: CN201620322979, 2016.

7. CRRC Zhuzhou Institute Co., Ltd, "Specification for cables on board rolling stock-Part 1: Standard wall insulation cables up to AC $3 \mathrm{kV}$," Zhuzhou,China, 2010. 